\title{
Analysis of current induced by long internal solitary waves in stratified ocean
}

\author{
Hui-Qin Zhang · Jia-Chun Li
}

Received: 5 February 2010 / Revised: 4 May 2010 / Accepted: 15 June 2010 / Published online: 3 September 2010

(C) The Chinese Society of Theoretical and Applied Mechanics and Springer-Verlag GmbH 2010

\begin{abstract}
An approximate theoretical expression for the current induced by long internal solitary waves is presented when the ocean is continuously or two-layer stratified. Particular attention is paid to characterizing velocity fields in terms of magnitude, flow components, and their temporal evolution/spatial distribution. For the two-layer case, the effects of the upper/lower layer depths and the relative layer density difference upon the induced current are further studied. The results show that the horizontal components are basically uniform in each layer with a shear at the interface. In contrast, the vertical counterparts vary monotonically in the direction of the water depth in each layer while they change sign across the interface or when the wave peak passes through. In addition, though the vertical components are generally one order of magnitude smaller than the horizontal ones, they can never be neglected in predicting the heave response of floating platforms in gravitationally neutral balance. Comparisons are made between the partial theoretical results and the observational field data. Future research directions regarding the internal wave induced flow field are also indicated.
\end{abstract}

Keywords Induced current - Internal solitary waves . Stratified flow . Two-layer fluid . Asymptotic analysis

The project was supported by the Ministry of Science and Technology (MST) of China and Chinese Academy of Sciences (CAS) (863) (2006AA09A103-4) and Knowledge Innovation Program (KJCX-YW-L02).

H.-Q. Zhang · J.-C. Li ( $\varangle)$

Laboratory of Environmental Mechanics,

Institute of Mechanics, Chinese Academy of Sciences,

Beijing 100190, China

e-mail: jcli05@imech.ac.cn

\section{Introduction}

Long internal solitary waves (LISW) of large amplitude now have received increasing attention since they carry huge momentum/energy and may pose a threat to offshore structures [1-3]. The US Navy has been investigating the motion of the internal pycnocline in oceans because of several losses of modern submarines [1]. In the north of the South China Sea, strong current of about $2 \mathrm{~m} / \mathrm{s}$ induced by a group of internal solitons has swung the tanker connected with drilling rig about $110^{\circ}$ in a few minutes [2]. An oceanographic program was conducted to measure near-surface currents to warn the drill-ship of large currents associated with approaching internal waves [4]. Other typical accident report includes Osborne and Burch [5], who thought that drilling rigs have to withstand the internal wave force. So much potential threat to oceanic structures like platforms and submarines described above indicates that the internal wave is an important factor in the process of drill operations and production in ocean engineering. The major concern in practical engineering is the possible loads exerted on compliant and floating offshore platforms, which may excite motions of hulls and vibrations of the connected equipment such as risers and cables [3]. This greatly promotes research interest in the behavior and characteristics of internal solitary waves (ISW) $[6,7]$.

It is well known that internal waves when passing through a marine structure seem to play a role of current due to their long period or large $K C$ number. However, there is only a dearth of studies concerning the wave induced current in contrast to the great deal of work on the internal wave itself. Even though the wave induced fluid velocity in the horizontal direction in two-layer case was derived from KdV theory, there is no detailed property analysis available so far [1]. By Roxana [8], the horizontal velocity field in the two-layer stratified ocean was constructed based on the conjugate state 
concept. Other typical work such as the fields of horizontal velocity of large amplitude internal solitary waves based on the Euler model for three water stratifications was given by Brand and Rubino [6]. Moreover, few work paid attention to the corresponding vertical components either theoretically or numerically. Nevertheless, their associated currents have profound implications in the design of ocean engineering to guarantee the safety of offshore structures. For example, current meters and thermistor chains were deployed in the proximity of a drill-ship over the continental shelf off Baffin Island to detect large currents associated with approaching internal waves, which displayed the undesirable effect of deflecting the dynamically positioned drill-ship [4]. As an additional example, the interaction between strong current on the Norwegian shelf slope and the pipelines of long spans along the uneven sea floor nearby the gas field Ormen Lange excited unexpected coupling vibration. The situation has promoted the development of various methods for forecasting subsea currents, loads and corresponding pipeline vibration [9].

Although the internal wave induced flow field is a crucial environmental factor in exploring the mechanism of interaction between internal waves and ocean platforms, the associated theory and modeling method in this regard are still not well developed. For this reason, particular attention in this paper is focused on this challenging issue, namely, the currents, induced by LISW with long wave length and period in ocean engineering.

\section{$2 \mathrm{KdV}$ equations, modal functions and flow fields}

We assume that the internal wave motion occurs in a twodimensional, inviscid and incompressible, density-stratified fluid bounded above by a free surface and below by a rigid wall. Suppose that the flow can be described in the coordinate system $(x, z)$ where $x$ is directed horizontally and $z$ vertically (see Fig. 1), so that the horizontal and vertical velocity components $(u, v)$, the fluid density $\rho$ as well as the pressure $p$ in Cartesian coordinate satisfy the continuity equation (1) and Euler equations (2)-(4),

$$
\begin{aligned}
& \frac{\partial u}{\partial x}+\frac{\partial v}{\partial z}=0 \\
& \frac{\partial \rho}{\partial t}+u \frac{\partial \rho}{\partial x}+v \frac{\partial \rho}{\partial z}=0 \\
& \rho\left(\frac{\partial u}{\partial t}+u \frac{\partial u}{\partial x}+v \frac{\partial u}{\partial z}\right)+\frac{\partial p}{\partial x}=0 \\
& \rho\left(\frac{\partial v}{\partial t}+u \frac{\partial v}{\partial x}+v \frac{\partial v}{\partial z}\right)+\frac{\partial p}{\partial z}+g \rho=0
\end{aligned}
$$

where $g$ is the gravity acceleration and $t$ is the time coordinate.

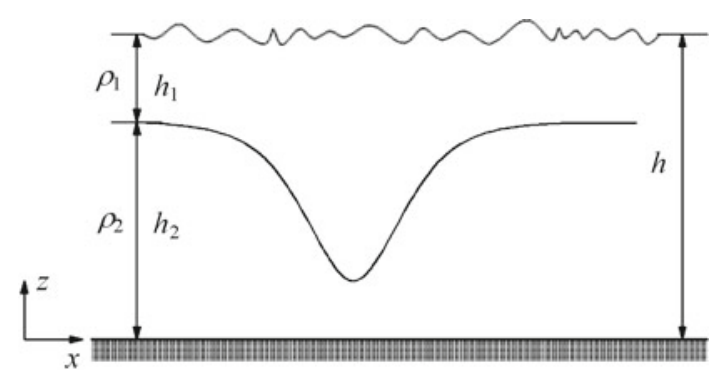

Fig. 1 Illustration of fluid coordinate system in which the upper boundary extends to the free surface and the lower to the rigid wall. Right panel stratification fluid. $h$ is the undisturbed water depth; left panel two-layer fluid. Let the density be a constant $\rho_{1}$ in the upper layer of depth $h_{1}$ and $\rho_{2}>\rho_{1}$ in the lower layer of depth $h_{2} \cdot h_{1}+h_{2}=h$

When the fluid is in the basic stable state, it has a density $\rho_{0}(z)$ satisfying $\mathrm{d} \rho_{0} / \mathrm{d} z<0$, a corresponding static pressure field $p_{0}(z)$ such that $\mathrm{d} p_{0} / \mathrm{d} z+\rho_{0} g=0$ and without flow in $x$ - and $z$-direction.

The formal derivation of the Sturm-Louiville equation for modal functions can be done through the introduction of a small parameter $\varepsilon \ll 1$, which characterizes the balance between the nonlinear wave-steepening effect and the linear wave dispersion to describe the long-wave approximation, and is defined as $\varepsilon=O(a / h)=O\left((h / L)^{2}\right)$. Here $a$ and $L$ are the wave amplitude and the wave length, respectively; $h$ is the undisturbed water depth. The detailed asymptotic analysis required may be found in Ref. [10], hence we shall only give a brief outline of the derivation.

Making the independent transformations for $x, t$, and $z$, and seeking asymptotic expansions for dependent variables $u$ and $v$ in Eqs. (1)-(4), respectively,

$\xi=\varepsilon^{1 / 2}(x-c t), \quad \tau=\varepsilon^{3 / 2} t, \quad z=z$,

$u=\varepsilon u_{1}+\varepsilon^{2} u_{2}$,

$v=\varepsilon^{1 / 2}\left(\varepsilon v_{1}+\varepsilon^{2} v_{2}\right)$,

where $c$ denotes the linear long wave speed.

Finally, we obtain the required Sturm-Louiville equation for $v_{1}$

$\frac{\mathrm{d}}{\mathrm{d} z}\left(\rho_{0} \frac{\mathrm{d} v_{1}}{\mathrm{~d} z}\right)-\frac{g}{c^{2}} \frac{\mathrm{d} \rho_{0}}{\mathrm{~d} z} v_{1}=0$,

along with the boundary condition at the sea bottom

$v_{1}=0, \quad$ at $z=0$,

as well as the combined kinematic and dynamic conditions at the undisturbed sea surface,

$\frac{\mathrm{d} v_{1}}{\mathrm{~d} z}-\frac{g}{c^{2}} v_{1}=0, \quad$ at $z=h$.

Equation (8), together with boundary conditions (9) and (10), constitutes a differential system which defines an 
eigenvalue problem for the vertical velocity component $v_{1}$ accurate to the first order.

Similarly, the Sturm-Liouville equation for the term of the second order in $\varepsilon$ can be obtained

$\frac{\mathrm{d}}{\mathrm{d} z}\left(\rho_{0} \frac{\mathrm{d} v_{2}}{\mathrm{~d} z}\right)-\frac{g}{c^{2}} \frac{\mathrm{d} \rho_{0}}{\mathrm{~d} z} v_{2}=F\left(v_{1}\right)$

$v_{2}=0, \quad$ at $z=0$,

$\frac{\mathrm{d} v_{2}}{\mathrm{~d} z}-\frac{g}{c^{2}} v_{2}=G\left(v_{1}\right), \quad$ at $z=h$,

where $F\left(v_{1}\right)$ and $G\left(v_{1}\right)$ are functions of $v_{1}$.

The system (11)-(13) can be solved only when the compatibility condition is satisfied and thus, we derive the governing equation for the isopycnal displacement $\eta$, namely the Korteweg-de Vries (KdV) equation

$A \eta_{\tau}+B \eta \eta_{\xi}+E \eta_{\xi \xi \xi}=0$

where

$A=\frac{4 g}{c^{2}} \int_{0}^{h} \rho_{0} \varphi \varphi^{\prime} \mathrm{d} z$

$B=-c \int_{0}^{h}\left[2 \rho_{0} \varphi^{\prime 3}+\frac{g}{c^{2}} \varphi^{2}\left(\rho^{\prime}{ }_{0} \varphi\right)^{\prime}-\varphi\left(\rho^{\prime}{ }_{0} \varphi \varphi^{\prime}\right)\right] \mathrm{d} z$,

$E=c \int_{0}^{h} \rho_{0} \varphi^{2} \mathrm{~d} z$

where $\varphi$ is a modal function of $z$ determined by solving Eqs. (17)-(19).

Now we turn to the task of obtaining approximate expressions of currents associated with approaching long internal waves. It is necessary in ocean engineering to take into account vertical component effects, and in view of the free surface kinematic condition (15) of order one in $\varepsilon$

$v_{1}=-c \frac{\partial \eta}{\partial \xi}+\varepsilon \frac{\partial \eta}{\partial \tau}+u \frac{\partial \eta}{\partial \xi}$.

Solutions of the vertical velocity component are then sought in the following form

$v_{1}=-c_{p} \frac{\mathrm{d} \eta}{\mathrm{d} \xi} \varphi$

where $c_{p}$ is the nonlinear wave speed. The modal function $\varphi(z)$ can be determined from the following equations derived by substituting Eq. (16) into Eqs. (8)-(10), i.e. SturmLiouville eigenvalue problem for $\varphi(z)$

$\frac{\mathrm{d}}{\mathrm{d} z}\left(\rho_{0} \frac{\mathrm{d} \varphi}{\mathrm{d} z}\right)-\frac{g}{c^{2}} \frac{\mathrm{d} \rho_{0}}{\mathrm{~d} z} \varphi=0$

$\varphi=0, \quad$ at $z=0$,

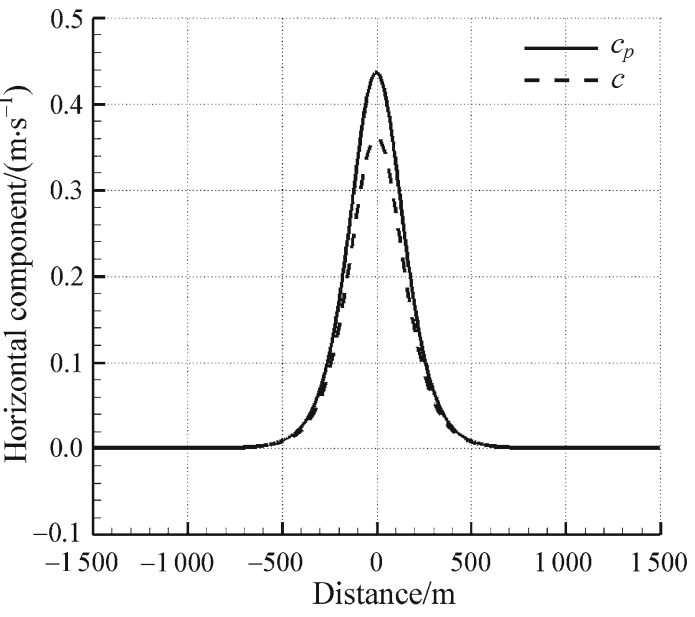

Fig. 2 Peak current speed at the crest of the wave. The solid line is the profile of the horizontal component in the direction of the wave propagation calculated from Eq. (21) or (28) based on the nonlinear wave speed $c_{p}$; the dashed line indicates the Su's result [10] based on the linear wave speed $c$ and calculated for the same case. The background stratification is taken as $\sigma=0.002, h_{1}=35 \mathrm{~m}, h_{2}=315 \mathrm{~m}$ and the wave amplitude is $45 \mathrm{~m}$

$\frac{\mathrm{d} \varphi}{\mathrm{d} z}-\frac{g}{c^{2}} \varphi=0, \quad$ at $z=h$.

Applying continuity equation (1) to transformations equations (5)-(7), the wave induced fluid velocity component in horizontal direction is obtained as

$u_{1}=c_{p} \eta \frac{\mathrm{d} \varphi}{\mathrm{d} z}$.

Returning Eqs. (16) and (20) to the initial variables $(u, v)$, we have

$u=\varepsilon c_{p} \eta \frac{\mathrm{d} \varphi}{\mathrm{d} z}$,

$v=-\varepsilon^{3 / 2} c_{p} \frac{\partial \eta}{\partial \xi} \phi$.

A remarkable difference between the induced velocity field system Eqs. (21)-(22) and the corresponding derivation in Ref. [10] is in their coefficient, i.e. the nonlinear wave speed $c_{p}$ replaces the linear long wave speed $c$, and the structure of the wave induced fluid velocity profile in the horizontal direction is consistent with those obtained previously by Grue and Trulsen [1]. The expressions for the nonlinear wave speed $c_{p}$ is the higher-order modification of $c$ valid to $O(\varepsilon)$, and the specific magnitude of horizontal component in twolayer fluid as an special case is illustrated in Fig. 2.

It is readily seen from Eqs. (21) and (22) that the velocity field is determined by the nonlinear wave speed $c_{p}$, the isopycnal displacement $\eta$ and the modal function $\varphi$. Considering $\eta$ can be obtained by $\mathrm{KdV}$ equation (14), the main point becomes how to determine $\varphi$. In general, the boundary value problem Eqs. (17)-(19) governing $\varphi$ can be readily solved 
numerically. On the other hand, it is also very helpful to seek some analytical solutions in certain special cases.

\section{Two-layer stratified fluid model}

A useful case in practical application is for a stratified ocean with sharp pycnocline of small thickness. As a result, a simplified two-layer model is roughly applicable for the study of the kinematic and dynamic processes. Assume a twolayer fluid of constant density $\rho_{1}$ and basic depth $h_{1}$ overlying another fluid of constant density $\rho_{2}$ and basic depth $h_{2}$ (see Fig. 1), that is

$\rho_{0}(z)=\rho_{1} H\left(z-h_{2}\right)+\rho_{2} H\left(-z+h_{2}\right)$,

where $H(z)$ is the Heaviside function.

For the two-layer fluid, the KdV equation (14) can be simplified as [11]

$\eta_{\tau}+\mu \eta \eta_{\xi}+\lambda \eta_{\xi \xi \xi}=0$,

where the coefficients $\mu$ and $\lambda$ turn out to be

$\mu=\frac{B}{A}=\frac{3}{2} c \frac{h_{1}-h_{2}}{h_{1} h_{2}}, \quad \lambda=\frac{E}{A}=\frac{c}{6} h_{1} h_{2}$

Evidently, Eq. (23) has the solitary wave solutions as follows

$\eta=a \cdot \operatorname{sech}^{2} \beta(\xi-V \tau)$,

where $a$ denotes the wave amplitude, and $V$ and $\beta$ are defined by

$V=\frac{1}{3} \mu a, \quad \beta=\sqrt{\frac{\mu a}{12 \lambda}}$.

Note that the actual total speed i.e. the nonlinear wave speed in two-layer fluid case is $c_{p}=c+\varepsilon V$. The characteristic half wave width $\beta^{-1}$ as a measurement of the solitary wave length is proportional to $|a|^{-1 / 2}$ and dependent on the coefficients of the nonlinear and dispersion terms $\mu$ and $\lambda$, which are further determined by the background stratification as illustrated in Eq. (24).

According to Sturm-Liouville eigenvalue problem Eqs. (17)-(19), the unique internal modal function in this case is given by (see Fig. 3)

$\varphi= \begin{cases}\frac{-z+\left(h_{1}+h_{2}\right)}{h_{1}}, & h_{2}<z<h_{1}+h_{2}, \\ \frac{z}{h_{2}}, & 0<z<h_{2},\end{cases}$

and

$c=\sqrt{\frac{g^{\prime} h_{1} h_{2}}{h_{1}+h_{2}}}$,

where $g^{\prime}$ is the so-called reduced gravity and can be expressed as $g^{\prime}=g \sigma$, and $\sigma$ is the relative layer density difference defined as $\sigma=2\left(\rho_{2}-\rho_{1}\right) /\left(\rho_{2}+\rho_{1}\right)$.

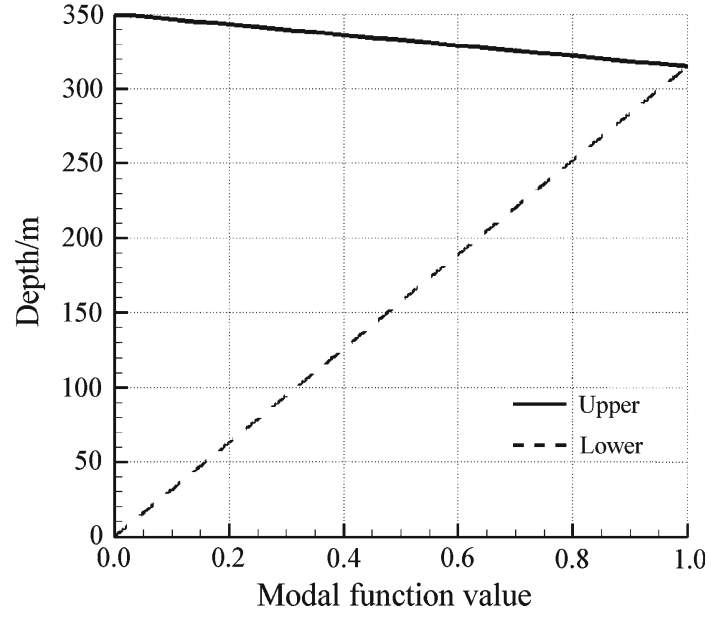

Fig. 3 Diagram of the internal modal function of two-layer system defined by Eq. (27) for $35 \mathrm{~m}$ upper layer depth and $315 \mathrm{~m}$ lower depth

After substitution of Eqs. (25) and (27) into Eqs. (21) and (22), the wave induced fluid velocity profile in two-layer case looks like, i.e. for $h_{2}<z<h_{1}+h_{2}$

$u=\varepsilon c_{p} a \cdot \operatorname{sech}^{2} \beta(\xi-V \tau)\left(-\frac{1}{h_{1}}\right)$,

$v=-\varepsilon^{3 / 2} c_{p}\left[-2 \beta a \cdot \operatorname{sech}^{2} \beta(\xi-V \tau) \tanh \beta(\xi-V \tau)\right]$

$$
\times\left(\frac{-z+h_{1}+h_{2}}{h_{1}}\right),
$$

and for $0<z<h_{2}$

$u=\varepsilon c_{p} a \cdot \operatorname{sech}^{2} \beta(\xi-V \tau)\left(\frac{1}{h_{2}}\right)$,

$v=-\varepsilon^{3 / 2} c_{p}\left[-2 \beta a \cdot \operatorname{sech}^{2} \beta(\xi-V \tau) \tanh \beta(\xi-V \tau)\right]$

$$
\times\left(\frac{z}{h_{2}}\right) \text {. }
$$

Then, we can derive some important properties of the induced flow velocity components in the two-layer stratified ocean case based on Eqs. (28) and (29).

The first components of Eqs. (28) and (29) follow from the fact that the induced horizontal flow velocity $u$ in the direction of propagation has the same profile as $\eta$, the departure of the interface from the mean position. Hence, the magnitude of the horizontal component is pulselike when long internal waves pass through. That is to say, the horizontal current experiences a process that from small to maximum and then to small, and the maximal current is achieved when the wave peak arrives. At the same time the magnitudes of the horizontal component in each layer are basically uniform with a shear at the interface and inversely proportional to the thickness of the corresponding layer, namely, the thinner the layer, the larger the induced current, and the maximum magnitude of the flow velocity component in the whole flow field is completely determined by the thinner layer thickness. 
Since the first and second term in Eqs. (28) and (29) are combined by continuity equation, respectively, thus the second component of Eqs. (28) and (29) i.e. the vertical components almost share the same analysis with the corresponding first term. We can find the induced vertical flow velocity

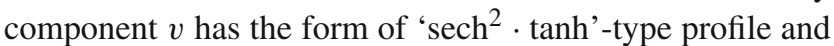
displays two peaks, one is positive and another one is negative in the horizontal direction. As horizontal components reach peaks at the wave crest or trough, the vertical counterparts vanish. On the other hand, we note that the vertical component varies linearly in the vertical direction in each layer with a maximum at the interface.

In addition, we should mention the influence of pycnocline strength in terms of the relative layer density difference. We can see from Eqs. (28) and (29) that the velocity components are proportional to the square root of the relative density difference and the total water depth. As you know, the former quantity for internal waves is usually 0.003 compared to 1.0 for surface waves, and the velocity components can thus be estimated around 0.05 of that for the corresponding components for surface waves.

\section{In-situ observations and model certification}

In order to more specifically verify and qualitatively understand the theoretical models (21) and (22) in continuous fluid, or Eqs. (28) and (29) in two layer case, we compare theoretical predictions with in situ observations. For this study, the measurements came from several current meters and a thermistor chain nearby a drill-ship operating at the site $\left(62^{\circ} 11^{\prime} \mathrm{N}, 62^{\circ} 59^{\prime} \mathrm{W}\right)$ in Davis Strait on the Atlantic coast of northern Canada by Cummins and Le Blond [4]. One of the reasons for this program was to warn the drill-ship of large currents associated with approaching internal waves. This observation work is distinguished by detailed measurements of hydrodynamics. The data collected by these instruments revealed the presence of internal waves from the coast, past the observation site, towards open water. The waves were characterized by a pulselike increase in current speed to a maximum and lasting from 10 to $15 \mathrm{~min}$. The maximum flow was observed at a depth of about $35-40 \mathrm{~m}$ and there was also another flow which had the opposite direction. The relative layer density difference $\sigma$ for the observation site can be calculated by using the profile of density anomaly $\left(\sigma_{t}\right)$ in Fig. 6a in Ref. [4], i.e. the upper layer 25.9 and the lower 27.5 and thus the magnitude of $\sigma$ is about $2.0 \times 10^{-3}$.

In this paper, based on the above observations, the parameters we will analyze are given in Table $1 . h$ is the depth of the bay; $d$ is the pycnocline location depth i.e. the upper layer depth $h_{1} ; \sigma$ is the relative layer density difference; $T$ is the current lasting period and $\eta$ is the maximum isotherm displacement at about $30-40 \mathrm{~m}$ depth.
Table 1 The geographical location parameters

\begin{tabular}{llllll}
\hline Parameter & $h(\mathrm{~m})$ & $d(\mathrm{~m})$ & $\sigma(\% \circ)$ & $T(\min )$ & $\eta(\mathrm{m})$ \\
\hline Data & 350 & $30,35,40$ & $2,3,4$ & $10-15$ & $33 \pm 12$ \\
\hline
\end{tabular}

Results based on these parameters: the wave amplitude $a=45 \mathrm{~m}$, the relative layer density difference $\sigma=2 \%$, the upper layer $h_{1}=35 \mathrm{~m}$ and lower layer $h_{2}=315 \mathrm{~m}$ are visualized in Figs. 4, 5, 6. We notice from Fig. 4a that the flow profile is characterized by a pulselike increase in current speed to a maximum $0.44 \mathrm{~m} / \mathrm{s}$ upwards at the interface in the upper layer, and the flow velocity component in lower layer has the opposite direction amounted almost to $0.05 \mathrm{~m} / \mathrm{s}$. At the same time, we find from Fig. $4 \mathrm{~b}$ that the vertical components change sign across the interface or when the wave peak passes through and similarly a positive pulselike amount to a maximum about $0.06 \mathrm{~m} / \mathrm{s}$, then decay sharply to another opposite pulselike with the same magnitude. Evidently, compared to the horizontal flow component (Fig. 4a), the vertical counterpart (Fig. 4b) is about one magnitude of order smaller than the horizontal one, which implies that the current induced by the long internal wave directs almost horizontally. Nevertheless, the most prominent feature of the vertical component is that it changes sign in the direction of the wave propagation. Thus, the fact that offshore structures can be exposed to an alternative vertical external loading environment when the internal solitary wave trains pass through should be a potential threat to structure fatigue. So the vertical velocity component often being neglected need reconsider seriously in the prediction of heave response of platform in gravitationally neutral balance. This further implies that an adequate physical model must include the vertical flow component.

Let us look at the solitary wave length now. We find that the so-called equivalent wavelength $L$ for internal solitary waves can be determined as

$L=4(\beta \sqrt{\varepsilon})^{-1}$,

where the inverse of $\beta$ defined by the second term of Eq. (26) is called the half characteristic wave width. Therefore, the wave length of internal solitary waves is proportional to dispersion and inversely proportional to nonlinearity. For the in-situ observation site and the internal solitary wave we selected, the magnitude of the equivalent wavelength $L$ is about $757.6 \mathrm{~m}$ (where the corresponding departure of the interface is approximately reduced to $7 \%$ of the wave amplitude), which happens to fall in the observational range 568$852 \mathrm{~m}$ estimated by

$L=c_{p} T$,

where $T$ denotes the observational current lasting period taken as 10 and 15 min from Ref. [4]. 


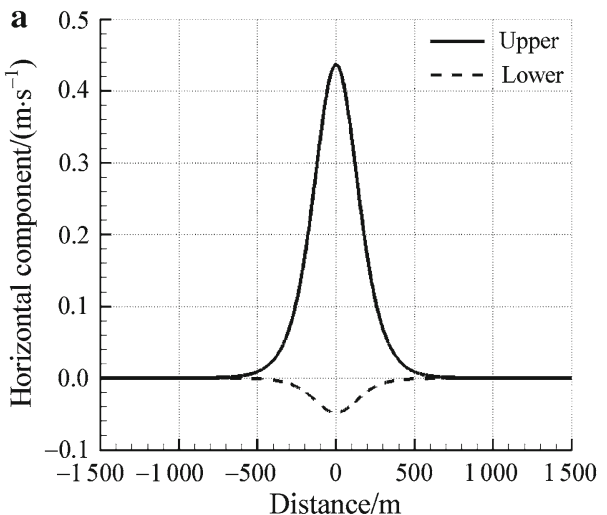

Fig. 4 Velocity components due to internal solitary waves obtained by Eqs. (28) and (29) for a horizontal calculated at the wave centre and b vertical calculated at the pycnocline. For a two-layer model, approximate theoretical flow velocity component profiles are plotted in the

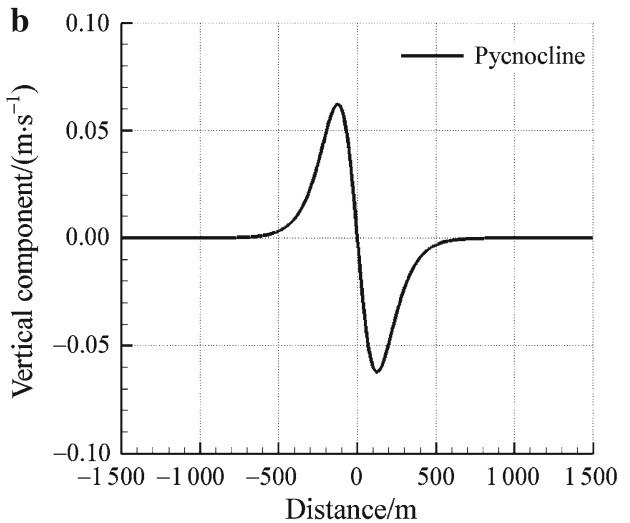

propagation direction of the internal solitary wave. The background stratification for calculation is the same as used in Fig. 3. The results show some obvious different features between the horizontal and vertical components in the flow velocity distribution
Fig. 5 Comparisons of horizontal velocity profiles calculated at the wave center in the direction of wave propagation. The comparison is done for a three upper/lower layer depths $h_{1}: h_{2}=30: 320,35: 315$ and $40: 310(\sigma=0.003)$ and $\mathbf{b}$ for three relative layer density differences $\sigma=0.002,0.003$ and $0.004\left(h_{1}: h_{2}=35: 315\right)$. The symbol upper and lower denotes the upper layer and lower layer, respectively

Fig. 6 Comparisons of the vertical velocity profile calculated at the pycnocline with $\sigma=0.003$ in the direction of wave propagation. The comparison is done for a three upper/lower layer depths $h_{1}: h_{2}=30: 320,35: 315$ and $40: 310(\sigma=0.003)$ and $\mathbf{b}$ for three relative layer density differences $\sigma=0.002,0.003$ and $0.004\left(h_{1}: h_{2}=35: 315\right)$
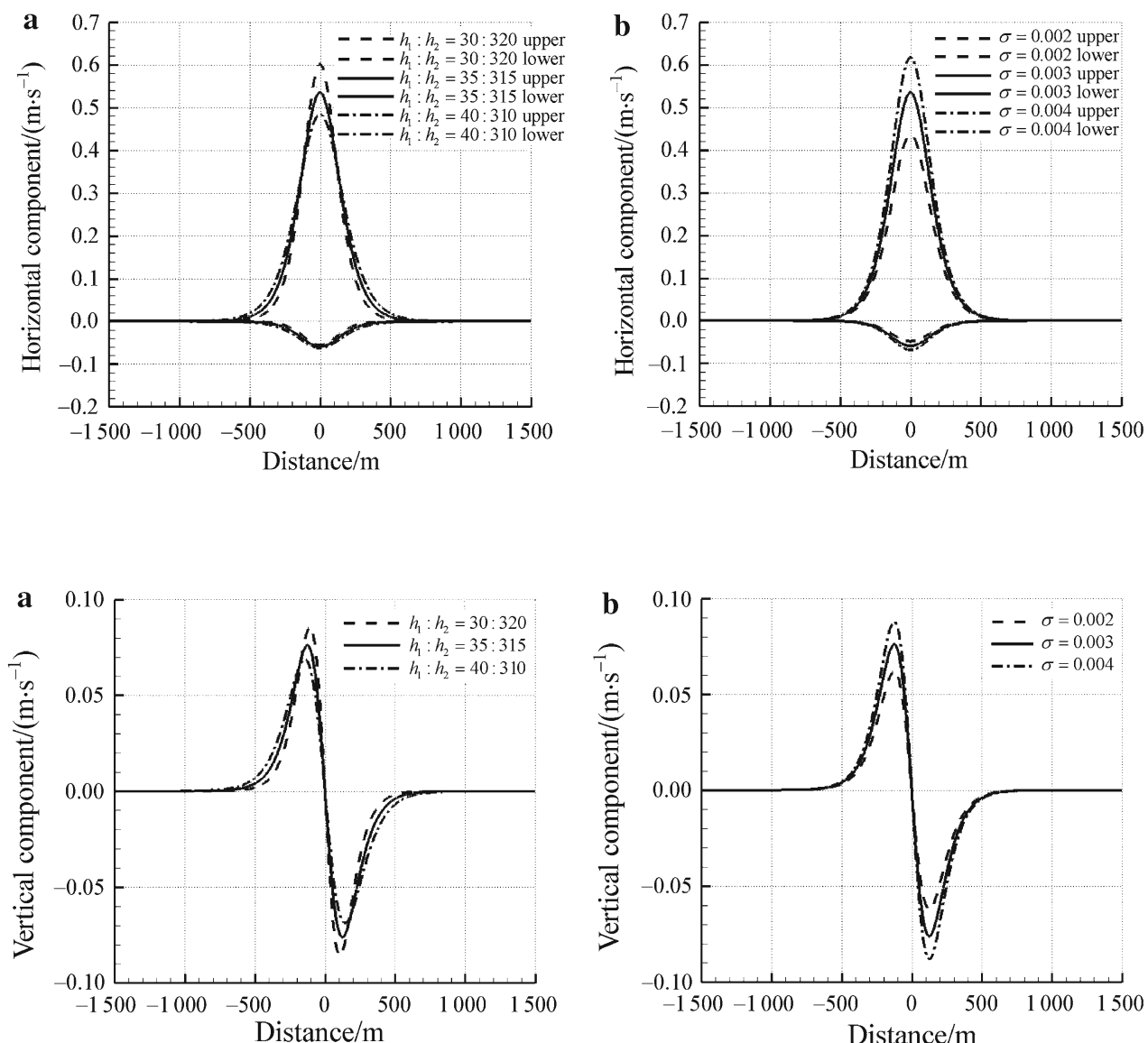

We have further made detailed comparison between the calculated results and the observational data to verify the theoretical model, which is listed in Table 2 for illustration. Here, $u_{m}$ is the peak current speed at the crest of the wave, $L$ is the equivalent wavelength which characterizes the horizontal length-scales, and $c_{p}$ is the phase speed. Satisfactory agreement between them confirms that the approximate theoretical scheme used for computing the peak current speed, the equivalent wavelength, and consequently the induced currents, is accurate and reliable.

Large-amplitude internal solitary waves, that is, their amplitudes and typical length scale of the vertical stratification are of the same order, are often observed $[12,13]$. As an example, computations are also performed with the 
Table 2 Comparisons of the parameters of currents induced by internal solitary waves between the theoretical and the observational results [4]

\begin{tabular}{llll}
\hline Parameter & Theoretical & \multicolumn{2}{l}{ Observational } \\
\cline { 3 - 4 } & & Mean & SD \\
\hline$\eta(\mathrm{m})$ & 45 & 33 & 12 \\
$u_{m}\left(\mathrm{~m} \mathrm{~s}^{-1}\right)$ & 0.437 & 0.44 & 0.15 \\
$c_{p}\left(\mathrm{~m} \mathrm{~s}^{-1}\right)$ & 0.95 & 1.09 & 0.11 \\
$L(\mathrm{~m})$ & 757.6 & & $568-852$ \\
\hline
\end{tabular}

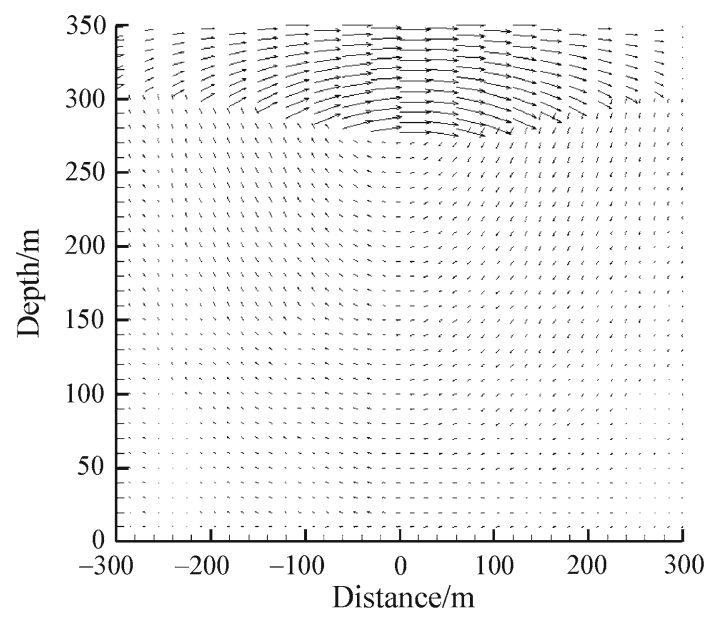

Fig. 7 Fields of currents associated with approaching internal waves obtained from Eqs. (28) and (29). The arrows in the upper layer show the current flow towards almost the direction of the wave propagation, and the dots in the lower layer indicate the opposite direction. It is visible from the vectors that the flow velocity magnitude in the upper layer is averagely larger than the counterpart in the lower layer

wave amplitude $100 \mathrm{~m}$ and the pycnocline $50 \mathrm{~m}$ in a water depth of $350 \mathrm{~m}$. We can obtain that the maximum flow velocity is about $1.7 \mathrm{~m} / \mathrm{s}$ at the wave peak and the opposite direction amounted to $0.3 \mathrm{~m} / \mathrm{s}$. For the vertical velocity component, the double magnitude can reach about $0.5 \mathrm{~m} / \mathrm{s}$ with an opposite sign. It is believed that the offshore platforms would face serious challenges when the internal waves induce such strong currents that pulselike impact forces are exerted on the offshore structures.

Figures 5 and 6 show the effects of horizontal and vertical velocities for three mean upper and lower layer depthes and three relative layer density differences. We can notice that the thinner the layer, the bigger the induced currents, while the induced currents increase with the relative layer density difference.

More visually, Fig. 7 further shows that the flow vector $(u, v)$ is relatively uniform on the upper and lower side of the interface, but exhibits strong shear nearby pycnocline with the maximum magnitude at the interface.

\section{Discussion and concluding remarks}

In the foregoing paragraphs, we have derived a theoretical expression for the velocity components and made a detailed analysis of the flow fields in the two-layer stratified fluid when a long internal solitary wave goes through. The theoretical results are well verified by in-situ field data acquired by several current meters and thermistor chains. As a result, we are able to come to the following conclusions:

The horizontal velocity components in the upper (lower) layer are characterized by a pulselike increase (decrease) to a positive maximum (negative minimum) in the direction of the wave propagation and independent of the water depth. This indicates that the horizontal components are basically uniform within each layer with a shear at the interface. In contrast, the vertical velocity counterpart depends linearly on the water depth with a maximum at the interface. Temporally, the vertical component profile is characterized by a double pulselike process, i.e. it has a positive pulse increase prior to the arrival of the wave peak, then decrease sharply to become null at the wave peak, and then exhibits a negative pulse, finally vanishes behind the approaching wave.

In the present article, we have provided a detailed analysis of flow fields induced by LISW in a two-layered stratified fluid. We note that the model is capable of providing a good estimate of flow field for the cases with a very thin pycnocline in-between the upper and lower layer, but fails to describe the structure of the velocity field otherwise. Therefore, this study would be made more complete by assessing the influence of different density stratifications.

Moreover, increasing attention has recently been paid to off-shore regions where the depth may reach as deep as $3,000 \mathrm{~m}$. The internal wave motion there is generally beyond the range of the KdV theory. Hence, further studies on the internal waves induced flow field should rely on the Benjamin-Ono theory often applied to the ocean with a shallow lighter layer water over a deeper heavier layer.

In the end, we know that the responses of the floating platform are of great concern in ocean engineering. With this motivation in mind, we hope the mechanisms underlying interactions between the internal solitary waves and the floating structures could be revealed based on our understanding on the current induced by LISW.

\section{References}

1. Grue, J., Trulsen, K.: Waves in Geophysical Fluids-Tsunamis, Rogue Waves, Internal Waves and Internal Tides. pp. 205-265. Springer, New York (2006)

2. Ebbesmeyer, C.C., Coomes, C.A., Hamilton, R.C., et al.: New observation on internal wave (solutions) in the South China Sea using an acoustic Doppler current profiler. In: Marine Technology Society 91 Proceedings, New Orleans, pp. 165-175 (1991) 
3. Zhang, H.Q., Li, J.C.: Wave loading on floating platforms by internal solitary waves. In: Proceedings of the ICFM-V, pp. 304-307, Shanghai, China (2007)

4. Cummins, P.F., Le Blond, P.H.: Analysis of internal solitary waves observed in Davis Straight. Atmos. Ocean. 22(2), 173-192 (1984)

5. Osborne, A.R., Burch, T.L.: Internal solitons in the Andaman Sea. Science 208, 451-460 (1980)

6. Vlasenko, V., Brand, P., Rubino, A.: Structures of large-amplitude internal solitary waves. J. Phys. Oceanogr. 30, 2172-2185 (1999)

7. Gaëlle, C., Alex, W.V., Thomas, F., et al.: An original method for characterizing internal waves. Ocean Model 31, 1-8 (2010)

8. Roxana, T.: Strongly nonlinear internal waves in near two-layer stratifications: generation, propagation and self-induced shear instabilities. Ph.D. Thesis, The University of North Carolina at Chapel Hill (2009)
9. Yttervik, R., Furnes, G.K.: Current measurements on the continental slope west of Norway in an area with a pronounced two-layer density profile. Deep Sea Res. I 52, 161-178 (2004)

10. Su, C.H.: Stratified Flow. Peking University Press, Beijing (1981) (in Chinese)

11. Grimshaw, R. : Internal solitary waves. In: Liu, P. L.-F. (ed.) Advances in Coastal and Ocean Engineering., pp. 1-30. World Scientific, NY (1997)

12. Stanton, T.P., Ostrovsky, L.A.: Observations of highly nonlinear internal solitons over the continental shelf. Geophys. Res. Lett. 25, 2695-2698 (1998)

13. Kropfli, R.A., Ostrovsky, L.A., Stanton, T.P., et al.: Relationships between strong internal waves in the coastal zone and their radar and radiometric signatures. J. Geophys. Res. 104, 3133-3148 (1999) 